\title{
Facile design and development of nano-clustery graphene-based macromolecular protein hydrogel loaded with ciprofloxacin to antibacterial improvement for the treatment of burn wound injury
}

\author{
Lipeng Zhu ${ }^{1} \cdot$ Linlu Chen ${ }^{1}$ (])
}

Received: 25 March 2021 / Revised: 25 June 2021 / Accepted: 27 August 2021 /

Published online: 21 September 2021

(c) The Author(s), under exclusive licence to Springer-Verlag GmbH Germany, part of Springer Nature 2021

\begin{abstract}
Nowadays, awareness about the burn wound is often considered difficult due to bacterial and other organism infections. The facile and eco-friendly preparations of antibiotic-loaded hydrogel-based bio-composites have great attention in the field of wound dressing for burn wound therapy and nursing care. In the present investigation, we have developed ciprofloxacin (CF)-encapsulated graphene-silk fibroin macromolecular hydrogel dressings material with unique chemical and physical properties to achieve the desirable antibacterial efficacy and healing activity. The antibacterial activity of prepared hydrogel was evaluated against bacterial pathogens treated with different concentrations of $\mathrm{CF}$, which have been provided improved antibacterial activity on burn wound infection. In vitro, cytocompatibility evaluations were performed to imply the suitability of hydrogel on fibroblast cells, which has been dramatically related to in vivo wound healing. Furthermore, an in vivo wound healing analysis was carried out using a rat to observe the capability of the CF-incorporated GH/SF hydrogel matrix. Thus, this investigation widely demonstrates the healing ability of prepared hydrogel matrix and could be a significant landmark in the research on burn wound healing applications.
\end{abstract}

Linlu Chen

chen.linlu@yahoo.com

1 Department of Burns, The First People's Hospital of Wenling, No. 333, Chuanan South Road, Chengxi street, Wenling 317500, People's Republic of China 


\section{Graphic abstract}

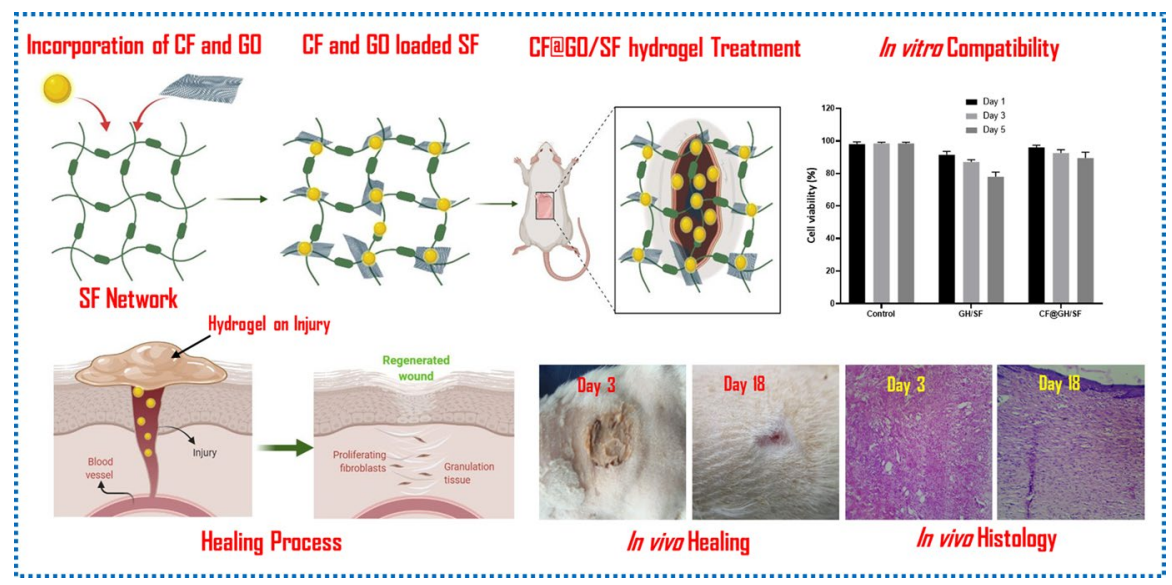

Keywords Burn wound - Graphene nano $\cdot$ Silk fibroin · Hydrogel · Ciprofloxacin · Antibacterial activity

\section{Introduction}

Burns is one of the worst forms of the outer layer of skin trauma and remains a serious global health issue. Burn wound is quickly susceptible to bacterial infection in skin tissue; it may delay the epidermal maturation and leads to surplus scar formation, organ failure, and death $[1,2]$. Inflammation, cell proliferation, and subsequent tissue formation are complex processes that the bacterial invasion mainly caused by Pseudomonas aeruginosa may delay the repairing and reconstructing burnt wounds [3]. To avoid these complications, we have to develop multifunctional antibiotics to protect the wound from an invasion of microorganisms to reduce infection, promote skin regeneration and tissue re-formation [4].

Several nontraditional antibacterial agents, such as metal, metal oxide NPs, carbon nanomaterials, and graphene-based materials, have been exploring in biomedical science. Among these materials, graphene-based materials have received plenty of attention for numerous applications, including biological that can provide a suitable scaffold for the wound healing process, which can improve the granulation tissue thickness, density of fibroblasts, collagen, and vascularization because of their stimulating effect on cell proliferation and electroconductivity properties [5-8]. Graphene is a building block for several carbon materials with no defect, and it consists of a single layer 2D structure. Therefore, graphene emerged as a promising material with its unique properties such as excellent mechanical strength, biocompatibility, hydrophilic groups, and surface area [9, 10]. The antibacterial and antifungal properties of graphene inhibit the wound from infection can potentially improve the wound healing process. Most promisingly, the graphene is used against corona virus 
porcine epidemic diarrhea when used a minor component of composite material [11]. Meanwhile, GO loading with active antibacterial substances shows superior antibacterial and dispersing effects [12]. On the other hand, physical damages of bacteria have been caused by graphene through direct contact of its sharp edges, and the chemical mechanism of graphene-attached cell membrane could induce oxidative stress $[9,13]$.

Natural polymers such as protein, peptides, carbohydrates, and polysaccharides have excellent biocompatibility that has been utilized for wound dressing. In particular, silk fibroin (SF) is a natural polymer that can activate the NF-кB signaling, closely relating to the cutaneous wound healing process. The excellent biocompatibility, improved mechanical property, non-cytotoxicity, low antigenicity, and noninflammatory properties of SF facilitate the enhanced cell adhesion, proliferation, tissue regeneration, and matrix deposition of keratinocytes and fibroblasts [14, 15]. Besides, it is very promising to control bacterial infection in different research studies and accelerated wound healing properties [16]. In addition, the degradation product of silk protein matched with body metabolism [17]. Various forms of silk fibroin have been used for wound dressing applications, including films, fiber, hydrogel, and sponges [18]. According to the characteristic structure of SF-based hydrogel with some unique properties such as adhesiveness and anti-oxidant to facilitate self-healing $[7,19]$, the connection with antibiotics is an initial stage of research for improving wound healing [20]. Antibiotics are generally used to prevent bacterial infection and biofilm formation on skin wounds. To date, many antibiotics have been reported to inhibit bacterial infections such as clindamycin, gentamicin, minocycline, and so forth [21-23]. Among the antibiotics, ciprofloxacin belongs to the fluoroquinolone family. It is one of the extensively used antibiotics for treating wound healing against in vitro bacterial infection; it's superior to other antibiotics in clinical practice, while their spontaneous bacterial resistance is a meager and lesser incidence of bacterial resistance $[24,25]$. Therefore, we need to fabricate the ciprofloxacin-loaded biodegradable hydrogel composite for wound dressing applications [26, 27].

Herein, we report the fabrication of CF-loaded nano-graphene/SF hydrogelbased biocomposite material an effective multifunctional antibacterial agent for burn wound treatment. The structural, morphological, and thermal properties were analyzed. The antibacterial efficiency of hydrogel-based biocomposite was evaluated. Finally, that synthesized hydrogel composites are effective for wound healing with enhanced antibacterial activity (CF) for preventing bacterial infection.

\section{Materials and methods}

\section{Materials}

Graphite flakes, sulfuric acid, hydrochloric acid, hydrogen peroxide, potassium permanganate, sodium carbonate dehydrous $\left(\mathrm{Na}_{2} \mathrm{CO}_{3}\right)$, lithium bromide $99 \%$, ciprofloxacin (CF), and ascorbic acid were purchased from Sinopharm Chemical Reagent Co., Ltd (Shanghai, China). Deionized water was used throughout the experiment. 


\section{Preparation of silk fibroin solution}

Silk fibroin was extracted according to the previous literature from Bombyx mori silk cocoons. First, the cocoons were boiled with aqueous $\mathrm{Na}_{2} \mathrm{CO}_{3}$ at $85{ }^{\circ} \mathrm{C}$ for removing sericin. After the obtained degummed silk, fibroin was dissolved in 9.3 $\mathrm{M} \mathrm{LiBr}$ solution at $60{ }^{\circ} \mathrm{C}$ for $2 \mathrm{~h}$. Then, the solution was dialyzed (MWCO 8000 ) against distilled water and centrifuged. The supernatant was stored at $4{ }^{\circ} \mathrm{C}$. Finally, the concentration of SF solution was measured at the weighing method [28].

\section{Preparation of graphene/silk fibroin}

Modified Hummer's method was applied to prepare graphene oxide (GO) from graphite flakes [29]. The dispersed graphene oxide was vigorously stirring for $30 \mathrm{mts}$ at $110{ }^{\circ} \mathrm{C}$ and sonicated for $1 \mathrm{~h}$ to get aqueous dispersion of GO. After that, ascorbic acid was added into the above mixture to get a proper reduction at $80{ }^{\circ} \mathrm{C}$ for $24 \mathrm{~h}$. Next, $100 \mathrm{mg}$ of silk fibroin (SF) was dissolving in $10 \mathrm{ml}$ phosphate buffer $(\mathrm{pH} 4)$ under constant stirring at $60{ }^{\circ} \mathrm{C}$ for $4 \mathrm{~h}$. Then, the prepared SF solution was then added into the above mixture with vigorous stirring for $30 \mathrm{mts}$ at $110^{\circ} \mathrm{C}$. The mixture was vortex until to get a homogeneous solution. Finally, the hydrogel was prepared by the ultrasonic method that the hydrogel formed by the self-assembly method. The final mixture was transferred into a beaker and degassed by ultrasound for $30 \mathrm{mts}$ and was regenerated using deionized water. The obtained black-colored hydrogel was thoroughly washed with water and dried.

\section{Preparation of CF-loaded nano-graphene/silk fibroin hydrogel}

$100 \mathrm{ml}$ of $\mathrm{CF}$ solution was added with graphene/silk fibroin solution placed in a water bath shaker for $3 \mathrm{~h}$ under dark conditions, and the supernatant solution was trained off. Finally, the CF-loaded graphene/silk protein composites were dried using a hot air oven and were characterized for further studies.

\section{Antibacterial activity}

The prepared hydrogel composites were tested by well diffusion method against pathogenic bacteria $S$. aureus-ATCC 11,632, and P. aeruginosa-ATCC 15,442. The inhibited zone of diameter around the disks was recorded of the test organism. The plates were autoclaved at $121{ }^{\circ} \mathrm{C}$ for $15 \mathrm{~min}$. Then, the agar medium was sterilized and transferred to a sterile petri dish. After getting solidified agar, the plates were stored at the refrigerator $\left(4^{\circ} \mathrm{C}\right)$. After freezing the media, the bacteria were diffused using a sterile cotton swab. The petri dishes were incubated for $24 \mathrm{~h}$ at $37^{\circ} \mathrm{C}$. After one day, the zone of inhibition of bacteria was measured. 


\section{Growth curve}

The antibacterial activity of composites was analyzed from bacterial growth inhibition by the standard microdilution method. The bacterial strain was grown in $10 \mathrm{~mL}$ of LB media-containing flask. Then, $0-40 \mu \mathrm{g} / \mathrm{mL}$ composites were supplemented with media, and the bacterial culture was incubated overnight under shaking environment at $160 \mathrm{rpm}$ in $37{ }^{\circ} \mathrm{C}$. The bacterial growth was determined by using $\mathrm{UV}-\mathrm{V}$ is spectrophotometer (OD $595 \mathrm{~nm}$ ) with a time interval. The growth curve was plotted against optical density versus time.

\section{Biofilm assay}

In biofilm inhibition assay, the biofilm formation was evaluated by CLSM (Carl Zeiss, LSM 710, Germany, software; Zen 2011) using 24-well plates, and bacterial viability was analyzed using Live/Dead Baclight bacteria viability kit. In brief, $100 \mu \mathrm{L}$ of bacterial cultures $\left(1 \times 10^{8}\right.$ cells $\left./ \mathrm{mL}\right)$ were incubated for $24 \mathrm{~h}$ in sterile LB broth with hydrogel. The planktonic cells and exhausted media were discarded after 24-h incubation. The non-adhering bacterial cells were removed using PBS, and each glass slide was stained with $0.01 \%$ acridine orange. The stained cell was detected using $488 \mathrm{~nm}$ wavelength for excitation and $540 \mathrm{~nm}$ for emission, and the images were observed under CLSM.

\section{MTT assay}

MTT (3-[4, 5-dimethyl-2-thiazolyl]-2, 5-diphenyl-2H-tetrazolium bromide) assay is used to determine the cell viability and cytotoxicity of the hydrogel. The cells were grown in 24-well plates at $37{ }^{\circ} \mathrm{C}$ in a humidified atmosphere of 3-5\% $\mathrm{CO}_{2}$ for $24 \mathrm{~h}$. After $24 \mathrm{~h}$ of incubation, the culture medium was discarded from each well, and then prepared hydrogels were transported into new 24-well culture plates (cell density $=2 \times 10^{4}$ cells per well). Then, the assays were incubated with MTT solution at $37{ }^{\circ} \mathrm{C}$ for $4 \mathrm{~h}$ with a tetrazolium salt solution. The MTT solution was discarded carefully, and $150 \mu \mathrm{L}$ of $10 \%$ dimethylsulfoxide (DMSO) was added into each well to solubilize the formazan crystal. The cell viability of hydrogel materials was evaluated at day 1, 3, and 5 after cell seeding. Each sample was analyzed under three replicates experiment for the exact quantitative value. Finally, the cell viability (\%) rate was measured by using the subsequent equation.

$$
\text { Percentage of cell viability }=[A]_{\text {Teated }} /[A]_{\text {Control }} \times 100
$$

\section{In vivo animal experiment}

In vivo animal experiments were approved and carried out according to the Department of Science and Education, Jinan People's Hospital, China. During this animal experiment, 5 weeks of Kunming mice were utilized. At first, each mice were anesthetized using controlled dosage and then $7 \mathrm{~mm}$ of partial thickness burns wounds 
were formed on back side of mice. After that, the hydrogel was applied in each model. The dressing samples were reformed on the wound every day. The healing rate was measured, and the photograph was taken at 3, 6, 12, and $18 \mathrm{~d}$. For histological examination during the wound regeneration, each model was stained with hematoxlin-eosin (H\&E) and photographed using a microscope.

\section{Characterization}

Thermogravimetric analysis of hydrogel composites was conducted on a Netzsch TG instrument under nitrogen atmosphere and the heating rate of $10{ }^{\circ} \mathrm{C} \mathrm{min}^{-1}$ in the temp range from room temp to $700{ }^{\circ} \mathrm{C}$. XPS was performed using an ESCALAB250Xi photoelectron spectrometer (ThermoFisher Scientific, USA) with X-ray radiation. The crystalline structure of hydrogel was examined by X-ray diffractometer (PAN analytical X'pert PRO) at $30 \mathrm{kV}$, and $40 \mathrm{~mA}$ was used as X-ray source. Raman spectra (LabRAM HR800, Horiba Jobin Yvon) were recorded to investigate the crystalline structure. SEM analysis was performed Carl Zeiss MA15/ EVO 18). FTIR spectra of hydrogel materials were recorded by Alfa Bruker FTIR Spectrophotometer.

\section{Result and discussion}

X-ray diffraction patterns of SF, graphene, SF/graphene, and hydrogel composites are shown in Fig. 1. The SF shows two peaks at $20^{\circ}$ and $24^{\circ}$, which are related to the $\beta$-sheet structure $[30,31]$. In graphene, the broad peaks appearing at around $2 \theta=25.5^{\circ}$ and $43.3^{\circ}$ are corresponding to (002) and (100) planes (Fig. 1b). The pattern was well matched with JCPDS NO: 75-1621 with hexagonal structure with sp2-bonded carbon atoms [32]. Meanwhile, the XRD patterns of the SF/
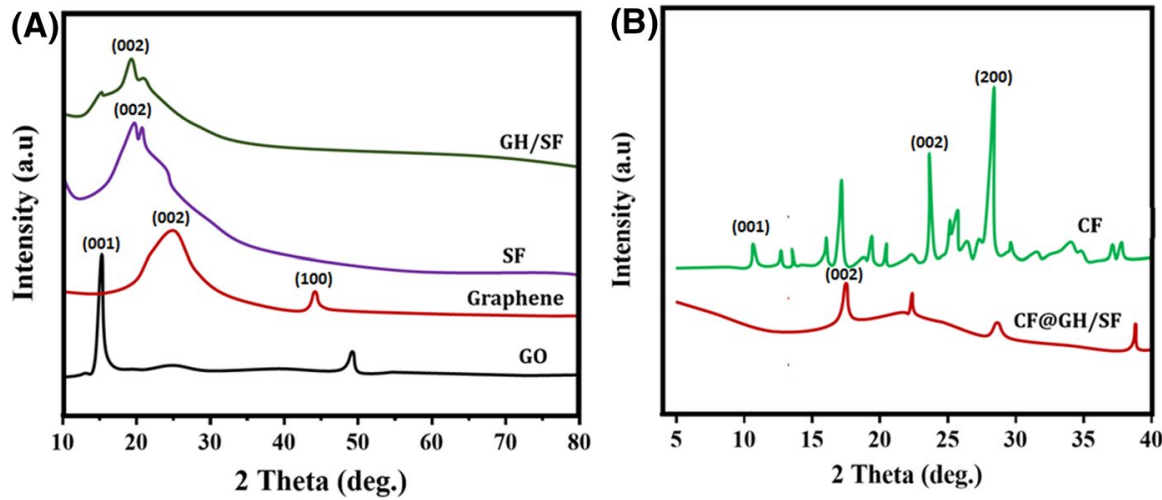

Fig. 1 The phase purity and crystallinity nature of the as-prepared samples were observed and exhibited XRD pattern analysis; a the results of graphene oxide (GO), bare graphene, silk fibroin (SF), and GOloaded SF sample (GO/SF); b the XRD patterns of bare ciprofloxacin (CF) and ciprofloxacin-encapsulated GO/SF (CF@GH/SF) samples 
graphene can be exhibited the characteristic peak of SF, which indicates that the addition GO does not affect the crystalline structure of SF due to the strong interaction between the SF and graphene [33]. Figure 1d shows the CF-loaded SF/ graphene displays a number of characteristic crystalline peaks is observed.

The morphology of SF, graphene, SF/graphene, and hydrogel composites was illustrated by SEM images and is presented in Fig. 2. The pure SF has depicted the interconnected macroporous structure, and a pore is distributed [31]. Graphene nanosheet consists of randomly aggregated, and cross-linked porous structure is shown in SEM images [29, 34]. The synthesized graphene/SF shows that the graphene sheet was robust and no exfoliation surface indicates that the nanosheet was well dispersed into a polymer matrix via polar-polar, H-bonding, and hydrophobic-hydrophobic interaction. As shown in Fig. 2b, a wrinkled film was covered on the fiber surface; at the same time, SF obtained a smooth surface without pleats [35].

The FTIR spectrum of SF, graphene, SF/graphene, and hydrogel composites is shown in Fig. 3a. In Fig. 3a, the characteristic peak at $3253 \mathrm{~cm}^{-1}, 1625 \mathrm{~cm}^{-1}$, $1522 \mathrm{~cm}^{-1}$, and $1234 \mathrm{~cm}^{-1}$ represents the $\mathrm{OH}$ stretching, amide I, amide II, and amide III of $\beta$-sheets and the random coil of SF [36, 37]. In Figure 3b, the characteristic bands of surface oxidized groups at $3450 \mathrm{~cm}^{-1}, 1384 \mathrm{~cm}^{-1}$, and $1028 \mathrm{~cm}^{-1}$ indicated the stretching vibration of $\mathrm{O}-\mathrm{H}, \mathrm{C}-\mathrm{O}-\mathrm{H}$, and $\mathrm{C}-\mathrm{O}$, respectively. The peak at $1635 \mathrm{~cm}^{-1}$ could be attributed to the skeletal vibration of graphitic carbon [34, 38]. For the SF/graphene, the spectra (Fig. 3c) presented the peaks of graphene at 1371 and $1589 \mathrm{~cm}^{-1}$ and the peak of SF at $1523 \mathrm{~cm}^{-1}$ and $1647 \mathrm{~cm}^{-1}$ strong, which revealed the successful hybridization of SF/graphene matrix [39]. The characteristic band at $1588 \mathrm{~cm}^{-1}$ and $1540 \mathrm{~cm}^{-1}$ from the benzene ring stretching vibration and the band at $3200 \mathrm{~cm}^{-1}$ and $1612 \mathrm{~cm}^{-1}$ are related to $\mathrm{O}-\mathrm{H}$ and $\mathrm{C}-\mathrm{O}$ stretching of CF-loaded SF/graphene [40]. Almost all the main peaks of the ciprofloxacin are detected into prepared hydrogel, indicating the successful formation of CF in hydrogel, and the peaks are shifted due to the intermolecular hydrogen bonding [41, 42].
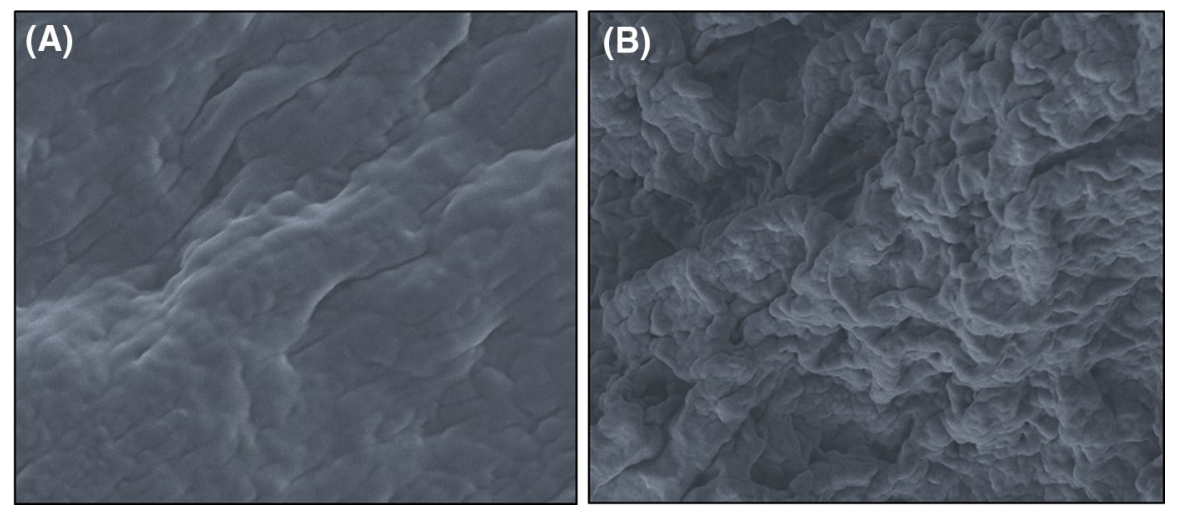

Fig. 2 The surface morphological structure of the prepared silk fibroin hydrogel in the absence (a) and presence (b) of nano-graphene sheet was exhibited through scanning electron microscopic (SEM) analysis 

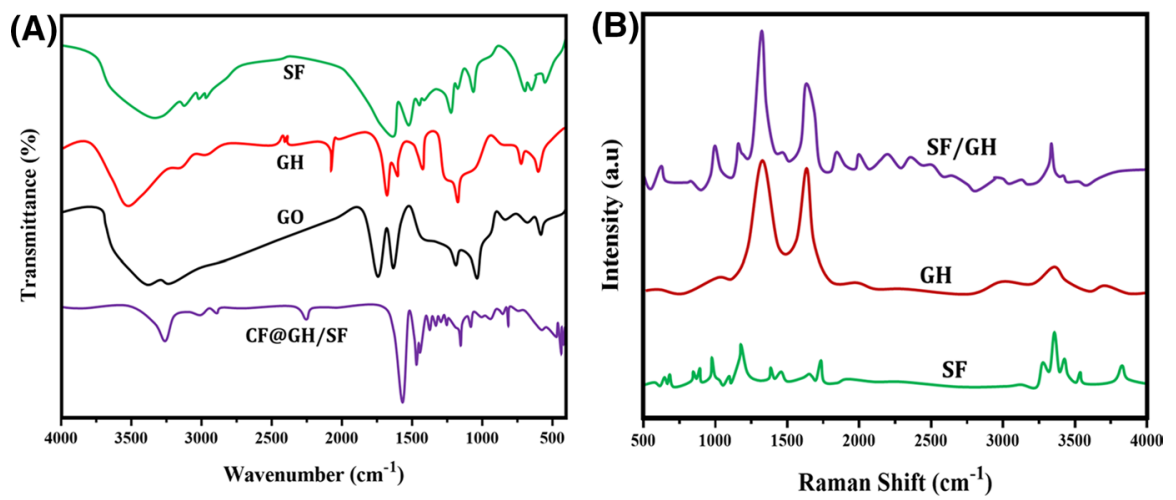

Fig. 3 The chemical structure and intermolecular interactions between the distributed nano-formulations and bio-polymeric groups have been observed and demonstrated by the FTIR and Raman spectroscopic analysis; a FTIR spectroscopic investigations of prepared SF, GH, GO and CF-loaded GH/SF samples and $\mathbf{b}$ Raman spectroscopic analysis of prepared SF, GH, and SF/GH, respectively

Raman spectra of graphene, SF, and SF/graphene hydrogel are shown in Fig. 3b. The Raman shift of SF was consistent with the spectral peaks around $1003 \mathrm{~cm}^{-1}$, $1326 \mathrm{~cm}^{-1}$, and $1658 \mathrm{~cm}^{-1}$, known as the characteristic $\beta$-structure [43]. The Raman spectra have prominent peaks observed at $1600 \mathrm{~cm}^{-1}$, attributed to the $\mathrm{G}$ band of carbon. The D band is an indication of structural defects or disorder of graphitic domains [29]. The ID/IG ratio is decreased, which denotes that the materials have a degree of disorder due to the introduction of SF in hydrogel [7, 44, 45]. As shown in Fig. 3b, SF/graphene hydrogel displays that the three dominant peaks at $1080 \mathrm{~cm}^{-1}$, $1225 \mathrm{~cm}^{-1}$, and $2943 \mathrm{~cm}^{-1}$ are attributed to the presence of SF [35]. Both the characteristic peaks of graphene and SF were visible from the distinct spectral line of the composites, which demonstrated that presence of graphene/SF.

To evaluate the thermal stability of graphene/SF composites as shown in Fig. 4, the first weight loss occurs at room temperature to $150{ }^{\circ} \mathrm{C}$, which is due to the removal of surface water that indicates the large amount of water bound in SF. After the addition of graphene, water is decreased gradually and reduced to $3.22 \mathrm{wt} \%$ for the graphene. All the samples showed that the stability is up to $160-210{ }^{\circ} \mathrm{C}$ while above the $210{ }^{\circ} \mathrm{C}$ the degradation is started. The rapid weight loss is observed in the pure silk sample, and the graphene has slight weight loss when the temp at $200{ }^{\circ} \mathrm{C}$ has higher thermal stability than other samples. Graphene is used in various materials with controlling thermal stability due to its excellent thermal properties [46]. The thermal stability of hydrogel increased when we used graphene into the hydrogel due to the hydrophobic interaction between graphene and SF [45].

The chemical composition of SF/graphene composites was carried out by XPS analysis. Figure 5 shows the characteristic peaks with binding energy $284.6 \mathrm{eV}$ and $533.3 \mathrm{eV}$, which is attributed to $\mathrm{C}(1 \mathrm{~s})$ and $\mathrm{O}(1 \mathrm{~s})$ [47]. In the spectrum of SF, the $\mathrm{C}$ $1 \mathrm{~s}, \mathrm{~N} 1 \mathrm{~s}$, and $\mathrm{O} 1 \mathrm{~s}$ peaks are centered in the binding energy of $287.1 \mathrm{eV}, 399.7 \mathrm{eV}$, and $532.7 \mathrm{eV}$ [48]. The peak is observed at $684.8 \mathrm{eV}$ in CF-loaded graphene/SF is evidence for F element existence in composites [49]. 


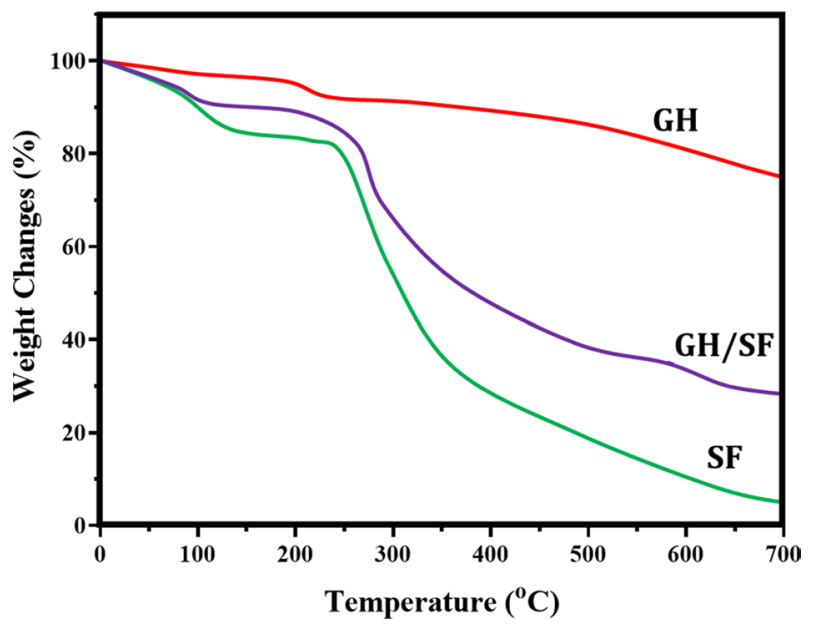

Fig. 4 The thermal stability and nature of temperature on prepared materials were observed by thermal gravimetric analysis (TGA); TGA results exhibited the thermal degradation behaviors of prepared SF, $\mathrm{GH}$, and $\mathrm{GH} / \mathrm{SF}$ in the temperature from 0 to $700{ }^{\circ} \mathrm{C}$

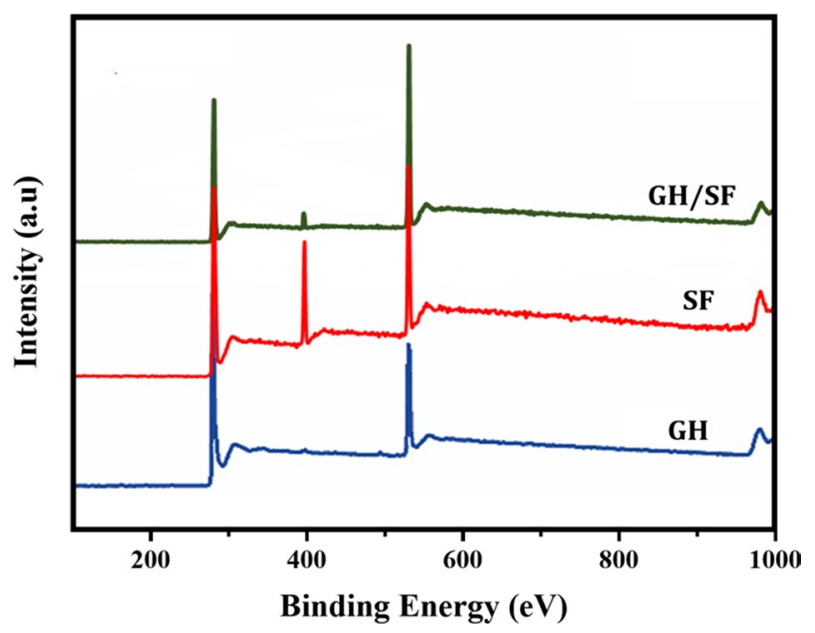

Fig. 5 The elemental presentations and nature of prepared hydrogel materials were observed by X-ray photoelectron spectrum (XPS) survey analysis; the presentation of carbon (C), nitrogen (N), and oxygen (O) elements into the prepared SF, GH, and GH/SF hydrogel samples was observed

The antibacterial activity of CF-loaded graphene/SF hydrogel was examined against $P$. aeruginosa and $S$. aureus is presented in Fig. 6 . The zone of inhibition method was used to evaluating the antibacterial properties as a simple and effective method. The wound healing can be invasively affected by P. aeruginosa, and $S$. aureus is the most common bacteria for creating a chronic wound. On the other hand, the great challenge of wound recovery directly depends on the intrinsic 

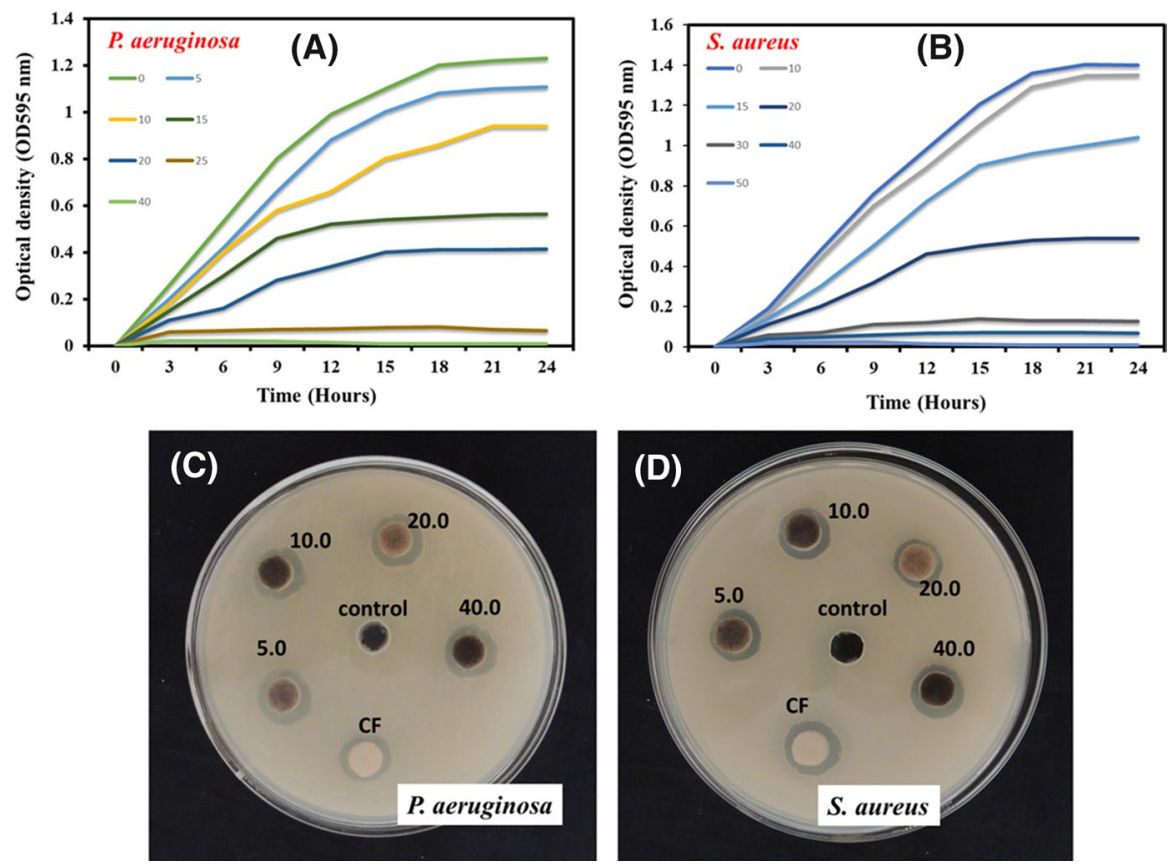

Fig. 6 Antibacterial properties of prepared CF-loaded GH/SF hydrogel materials were investigated against clinically approved gram-positive $(S$. aureus) and gram-negative (E. coli) bacterial pathogens; a \& b growth curve analysis of as-prepared CF@GH/SF hydrogel material in different concentrations and zone of inhibition; $\mathbf{c} \& \mathbf{d}$ results observation of prepared $\mathrm{CF} @ \mathrm{GH} / \mathrm{SF}$ sample in different concentration

antibiotic resistance of bacteria. Therefore, some powerful antibacterial agents can have used in biomaterials, particularly in wound dressing. The CF-loaded graphene/ SF exhibited distinctive antibacterial effects against $P$. aeruginosa and $s$. aureus with a clear zone of diameter as one of the important infectious microorganisms in wounds. However, low inhibition zone was formed around hydrogel without CF. These results showed the physical disruption of ROS species and sharp edges of GO which lead to remarkable damages [50]. These results suggested that the hydrogel has less zone of inhibition of $P$. aeruginosa and $S$. aureus, while CF-loaded hydrogel can effectively inhibit the growth and replication of $P$. aeruginosa and $S$. aureus because CF implies good release and diffuse out into the surface. These synthesized hydrogel composites have been used as an antibacterial drug delivery system with the controlled release [49].

Figure 6 shows the optical densities of composites and CF-loaded graphene/SF with various dose concentrations $\left(0-40 \mu \mathrm{g} \mathrm{mL}^{-1}\right)$ of $\mathrm{CF}$ were plotted at regular time intervals. The composites showed concentration-dependent relative rate and extent of bactericidal activity. The hydrogel composites exhibit both bacteriostatic and bactericidal activity because of their better efficiency, and there is a sudden decrease of growth of the bacteria when the concentration is increased therefore avoiding the exogenous infection effectively. Moreover, the antibacterial activity of composites is increased when the amount of CF-loaded graphene/SF is increased, owing to the 
sustained release of CF from composites and sustained antibacterial activity, and the hydrogel could play a reservoir. Therefore, we give proper protection against wounds when the treatment with burn wound dressing [51]. The present study reports that CF-loaded graphene/SF inhibits the bacterial growth in burn wounds from being exogenous infected for several days. Its acts as an effective source of antibacterial biocides.

The cytocompatibility of CF-loaded graphene/SF was investigated against human fibroblast cell line as exhibited in Fig. 7. Generally, the fibroblast cell line is a widely tested cell line for wound healing materials. It is commonly presented human cell lines and incredibly wound healing contributed cell lines in the skincare and wound management processes. The cell migration ability of the hydrogel has been exhibited at different time intervals, as shown in Fig. 7a. In addition, the signaling from the growth factors of fibroblast cells is the main factor of wound ECM synthesis and intracellular mechanisms of skin wound healing biological processes. In the present investigation, the fibroblast cell is treated with hydrogel nanocomposites, enhancing cell survival and proliferation at different time intervals. Figure $7 \mathrm{~b}$ shows the enhanced cell survival and proliferation (Fig. 7c) treated with hydrogel composites, which imply that the material is significantly influencing the inhibition of bacterial infection and improving cell viability. The above results showed that the enhanced proliferation is the combination of graphene; it may increase oxygen-containing groups [33].

In the normal wound healing process, a traditional dressing method could be suitable and adheres to the injured tissues and creates solutions to form newly produced
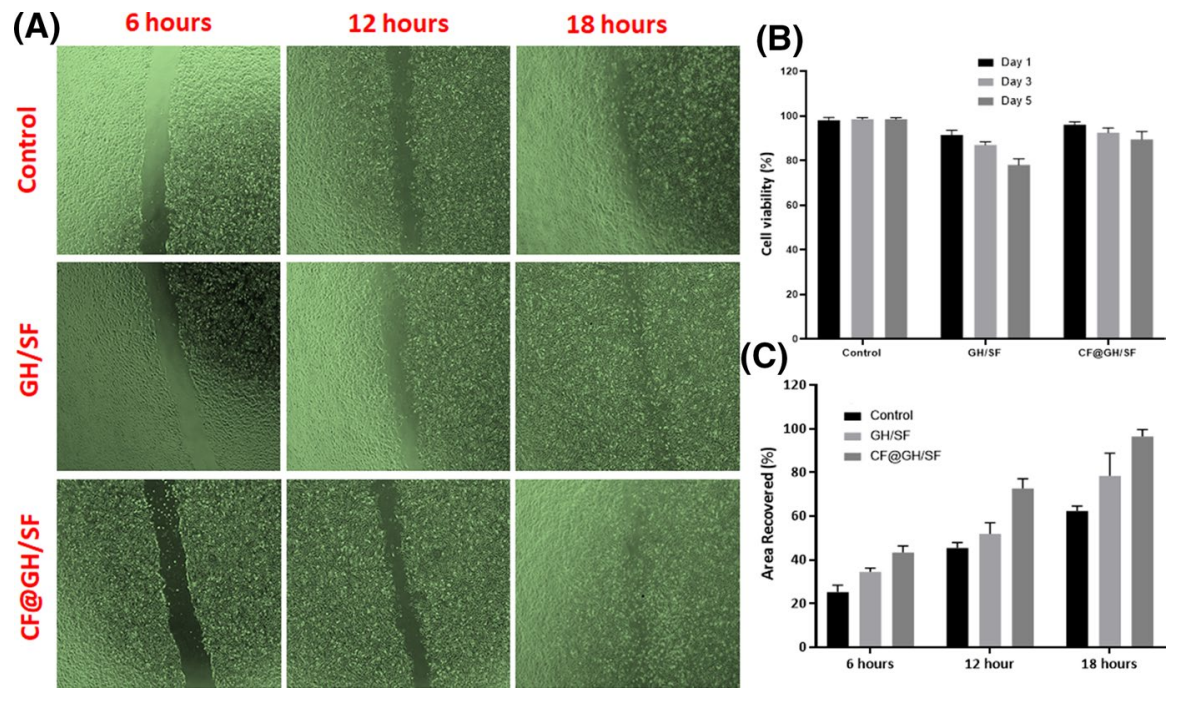

Fig. 7 In vitro cytocompatibility of prepared hydrogel materials was studied by the methods of quantitative observation of in vitro cell viability (MTT assay) and cell migration analysis in different time intervals; a microscopic visualization of in vitro cell migrations treated with control (PBS), GH/SF, and CF@ GH/SF samples in different time durations $(6,12$, and $18 \mathrm{~h}) ; \mathbf{b} \& \mathbf{c}$ qualitative results of cell viability and cell proliferation in different time intervals 
granulated tissues for the healing. But, the burn wound and other problematic wound healing processes need special attention and tailored materials compositions to provide superior properties such as antibacterial, anti-fouling, anti-oxidant etc., Furthermore, the anti-infectious ability of prepared CF-loaded GF/SF hydrogel matrix has been confirmed by the antibacterial experiments. In addition, in vivo healing ability of the prepared GF/SF and CF@GF/SF hydrogels was investigated on the backside of rats under post-treatment on different days (3, 6, and 12 days) as exhibited in Fig. 8. The healing ability on treated wounds was recorded about the total area covered (healed) in different post-treatment days. On post-treatment day 3, the wound environments were consistent and haven't observed many changes in all the groups. On post-treatment day 6, it was exhibited that the wound healing percentage of CF@ GF/SF and GF/SF hydrogels treated wound site have been higher and extensively covered the injured area when compared to the control samples. On post-treatment day 12, the wound site of GF/SF and CF@GF/SF hydrogel samples was significantly healed and covered the injured area compared to the control sample. These results showed that the antibacterial hydrogel could provide a suitable scaffold for cell attachment, proliferation, and wounds inhibition from infection can potentially improve the wound healing process [7]. The superior healing properties of hydrogel might be attributed to graphene and the sustained release of CF. The CF has excellent antibacterial and anti-inflammatory activity, which could effectively inhibit the bacteria infection on the wound surface, thereby accelerating wound healing [52]. The H\&E stained analysis has further evaluated the post-treated wound tissues on

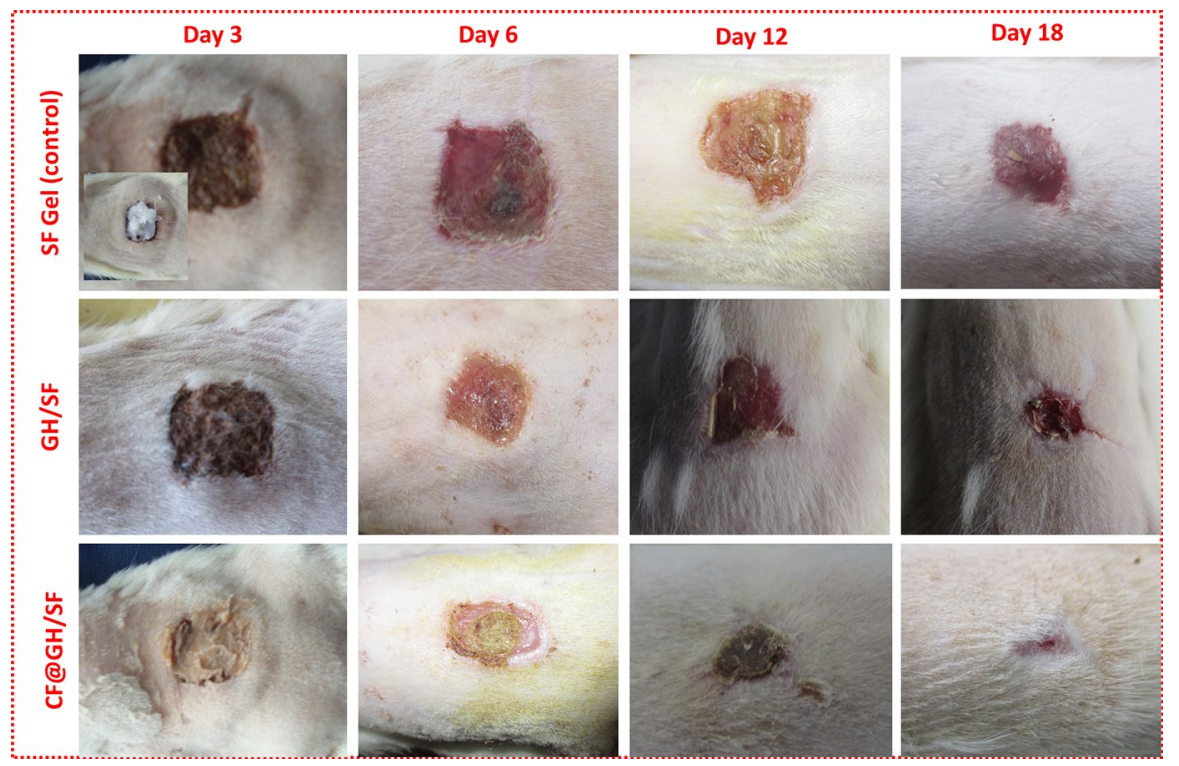

Fig. 8 In vivo burn wound healing was observed primarily by created burn wound injury and treated with prepared control (SF gel), GH/SF, and CF@GH/SF hydrogel samples in different postoperative days (3, 6, 12, and 18 days); The photovisualization exhibited that CF@GH/SF hydrogel sample has great healing ability when compared to GH/SF and control sample 
different days through microscopic analysis to observe its host immune responses, as shown in Fig. 9. The control sample shows more inflammatory cells on post-treatment days 6 and 12 when compared to the hydrogel samples, which was confirmed that prepared hydrogel samples provided significant anti-inflammatory response and host immune responses in the healing process. As shown in Fig. 9, after treatment for 12 days, CF@GF/SF hydrogel-treated wound site shows a clear dermis layer, which confirming that successful re-epithelialization when compared to the control and GF/SF hydrogel sample. These results established that CF@GF/SF hydrogel could promote the cell growth of fibroblasts, keratinocytes, and endothelial cells and showed a positive effect for the wound healing process during in vivo wound healing treatment [53]. The superior healing capacity of hydrogel might be attributed to the presence of GO, which promoted cellular proliferation and migration involved in the proliferative phase [52].

\section{Conclusion}

In summary, we report a synthesized graphene/silk fibroin hydrogel wound dressing material which was further loaded with ciprofloxacin and was characterized. The XRD study explored the synthesis of graphene using graphite powder. The functional group and structural properties are confirmed by FTIR and Raman spectra. The SEM images showed that the nanosheet was well dispersed into the polymer matrix. These composites hydrogel showed enhanced antibacterial activity toward

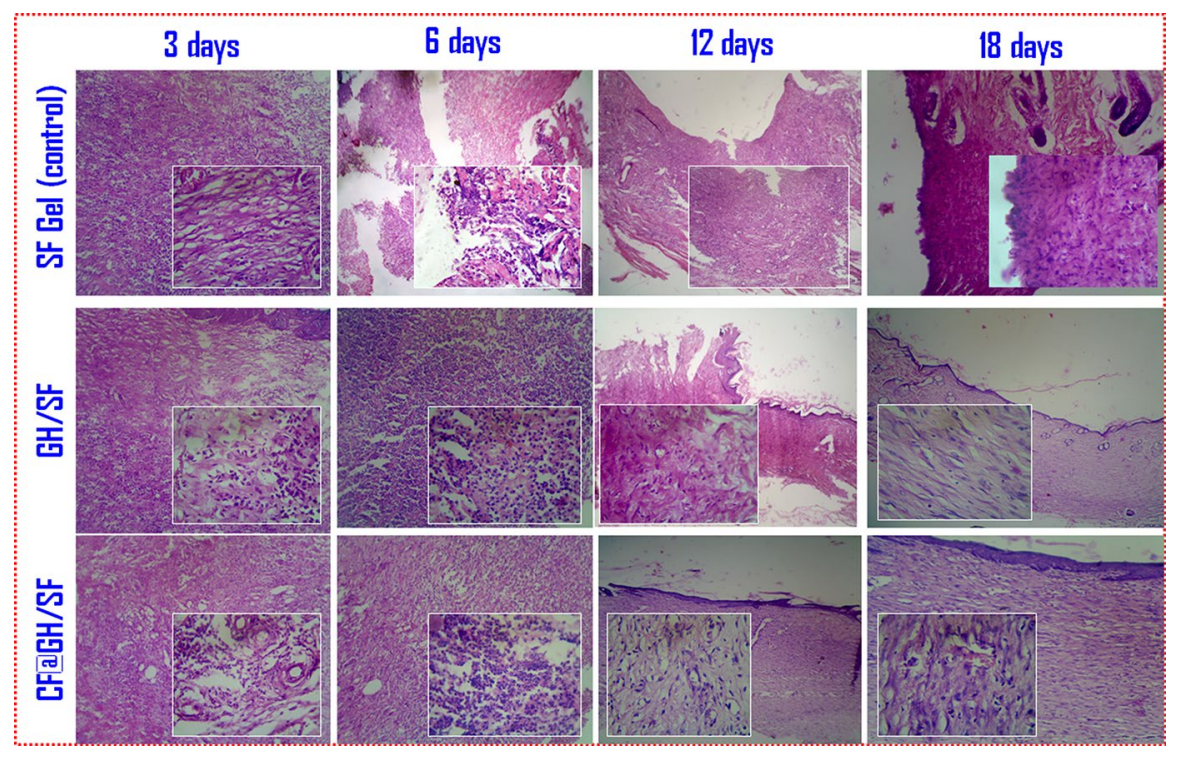

Fig. 9 Histomorphological observation (hematoxylin-eosin staining microscopic study) of burn wound regeneration after treatment of prepared hydrogel dressings of GH/SF, CG@GH/SF, and control sample on the $3 \mathrm{rd}, 6 \mathrm{th}, 12 \mathrm{th}$, and 18th day after postoperative; the images exhibited in different magnifications (10x and 40x) 
both bacteria. In vitro cytocompatibility studies confirmed that the hydrogel was nontoxic toward the fibroblast cell line. In addition, that the in vivo wound healing analysis of hydrogel effectively hastened the wound healing process after burning wound infections. In vitro and in vivo studies have been demonstrated the probable effect on fibroblast adhesion, fastening re-epithelialization, and keratinocyte proliferation. Overall, the CF-loaded graphene/silk protein hydrogel gave significant potential application; it is essential to wound dressing material in the burn healing process.

Acknowledgements Authors are heartfully acknowledging to Heilongjiang Youth Science Foundation (QC2016101), Harbin Science and Technology Innovation Talents Research Foundation (2016 RAQXJ159, 2016 RAXYJ069), and Heilongjiang Provincial Health and Family Planning Commission Research Project (2018-082).

\section{References}

1. Hashmi MU, Khan F, Khalid N, Shahid AA, Javed A, Alam T, Jalal N, Hayat MQ, Abbas SR, Janjua HA (2017) Hydrogels incorporated with silver nanocolloids prepared from anti-oxidant rich Aerva javanica as disruptive agents against burn wound infections. Coll Surf A Physicochem Eng Asp 529:475-486

2. Hill DM, Sinclair S, Hickerson W (2017) Rational selection and use of antimicrobials in patients with burn injuries. Clin Plast Surg 44:521-534

3. Rahimi M, Ahmadi R, Kafil HS, Shafiei-Irannejad V (2019) A novel bioactive quaternized chitosan and its silver-containing nanocomposites as a potent antimicrobial wound dressing: structural and biological properties. Mater Sci Eng C 101:360-369

4. Li W, Qianqian Yu, Yao H, Zhu Y, Topham PD, Yue K, Ren Li, Wang L (2019) Superhydrophobic hierarchical fiber/bead composite membranes for efficient treatment of burns. Acta Biomater 92:60-70

5. Sun Y, Yang Y, Yang M, Fei Yu, Ma J (2019) Response surface methodological evaluation and optimization for adsorption removal of ciprofloxacin onto graphene hydrogel. J Mol Liq 284:124-130

6. Faraji S, Nowroozi N, Nouralishahi A, Shayeh JS (2020) Electrospun poly-caprolactone/graphene oxide/quercetin nanofibrous scaffold for wound dressing: evaluation of biological and structural properties. Life Sci 257:118062

7. Zhang H, Zheng S, Chen C, Zhang D (2021) A graphene hybrid supramolecular hydrogel with high stretchability, self-healable and photothermally responsive properties for wound healing. RSC Adv 11:6367

8. Choudhary P, Ramalingam B, Das SK (2020) Fabrication of chitosan-reinforced multifunctional graphene nanocomposite as antibacterial scaffolds for hemorrhage control and wound-healing application. ACS Biomater Sci Eng 6(10):5911-5929

9. Ahmed MK, Afifi M, Awwad NS, Ibrahium HA (2020) Pb(II) and Cd(II) removal, mechanical and morphological features of nanofibrous membranes of cellulose acetate containing fillers of hydroxyapatite, graphene oxide, and magnetite. Appl Phys A 126:819

10. Fathi AM, Ahmed MK, Afifi M, Menazea AA, Uskokovic V (2021) Taking hydroxyapatitecoated titanium implants two steps forward: surface modification using graphene mesolayers and a hydroxyapatite-reinforced polymeric scaffold. ACS Biomater Sci Eng 7(1):360-372

11. Ahmed MK, Afifi M, Uskokovi V (2020) Protecting healthcare workers during COVID-19 pandemic with nanotechnology: a protocol for a new device from Egypt. J Infect Public Health 13:1243-1246

12. Jian Z, Wang H, Liu M, Chen S, Wang Z, Qian W, Luo G, Xia H (2020) Polyurethane-modified graphene oxide composite bilayer wound dressing with long-lasting antibacterial effect. Mater Sci Eng C 111:110833

13. Kumar P, Huo P, Zhang R, Liu Bo (2019) Antibacterial properties of graphene-based nanomaterials. Nanomaterials 9:737. https://doi.org/10.3390/nano9050737 
14. Mehrabani MG, Karimian R, Mehramouz B, Rahimi M, Kafil HS (2018) Preparation of biocompatible and biodegradable silk fibroin/chitin/silver nanoparticles 3D scaffolds as a bandage for antimicrobial wound dressing. Int J Biol Macromol 114:961-971

15. Liua J, Yana L, Yang W, Lan Y, Zhu Q, Hongjie Xu, Zheng C, Guo R (2019) Controlled-release neurotensin-loaded silk fibroin dressings improve wound healing in diabetic rat model. Bioact Mater 4:151-159

16. Eivazzadeh-Keihan R, Aliabadi HAM, Radinekiyan F, Sobhani M, Khalili F, Maleki A, Madanchi H, Mahdavi M, Shalan AE (2021) Investigation of the biological activity, mechanical properties and wound healing application of a novel scaffold based on lignin-agarose hydrogel and silk fibroin embedded zinc chromite nanoparticles. RSC Adv 11:17914

17. Feng Y, Li X, Zhang Q, Yan S, Guo Y, Li M, You R (2019) Mechanically robust and flexible silk protein/polysaccharide composite sponges for wound dressing. Carbohyd Polym 216:17-24

18. Gogurla N, Sinha AK, Naskar D, Kundu SC, Ray SK (2016) Metal nanoparticles triggered persistent negative photoconductivity in silk protein hydrogels. Nanoscale 8:7695

19. Yu R, Yang Y, He J, Li M, Guo B (2021) Novel supramolecular self-healing silk fibroin-based hydrogel via host-guest interaction as wound dressing to enhance wound healing. Chem Eng J 417:128278

20. Altoe LS, Alves RS, Sarandy MM, Morais-Santos M, Novaes RD, Goncalves RV (2019) Does antibiotic use accelerate or retard cutaneous repair? A systematic review in animal models. PLoS One. https://doi.org/10.1371/journal.pone.0223511

21. Sadeghi S, Nourmohammadi J, Ghaee A, Soleimani N (2020) Carboxymethyl cellulose-human hair keratin hydrogel with controlled clindamycin release as antibacterial wound dressing. Int J Biol Macromol 147:1239-1247

22. Bakhsheshi-Rad HR, Hadisi Z, Ismail AF, Aziz M, Akbari M, Berto F, Chen XB (2020) In vitro and in vivo evaluation of chitosan-alginate/gentamicin wound dressing nanofibrous with high antibacterial performance. Polym Test 82:106298

23. Mohebali A, Abdouss M, Taromi FA (2020) Fabrication of biocompatible antibacterial nanowafers based on HNT/PVA nanocomposites loaded with minocycline for burn wound dressing. Mater Sci Eng C 110:110685

24. Evranos B, Aycan D, Alemdar N (2019) Production of ciprofloxacin loaded chitosan/gelatin/bone ash wound dressing with improved mechanical properties. Carbohydr Polym 222:115007

25. Ajmal G, Bonde GV, Mittal P, Khan G, Pandey VK, Bakade BV, Mishra B (2019) Biomimetic PCLgelatin based nanofibers loaded with ciprofloxacin hydrochloride and quercetin: a potential antibacterial and anti-oxidant dressing material for accelerated healing of a full thickness wound. Int J Pharm 567:118480

26. Dillen K, Vandervoort Jo, Van den Mooter G, Ludwig A (2016) Evaluation of ciprofloxacin-loaded Eudragit@ RS100 or RL100/PLGA nanoparticles. Int J Pharm 314:72-82

27. Günday N, Torge A, Juntke J, Schwarz BC, Schneider-Daum N, Türeli AE, Lehr CM, Schneider M (2017) Ciprofloxacin-loaded PLGA nanoparticles against cystic fibrosis P. aeruginosa lung infections. Eur J Pharm Biopharm. https://doi.org/10.1016/j.ejpb.2017.04.032

28. Gong D, Lin Q, Shao Z, Chen X, Yang Y (2020) Preparing 3D-printable silk fibroin hydrogels with robustness by a two-step crosslinking method. RSC Adv 10:27225

29. Huang L, Li C, Yuan W, Shi G (2013) Strong composite films with layered structures prepared by casting silk fibroin-graphene oxide hydrogels. Nanoscale 5:3780-3786

30. Kumar RM, Rao BL, Asha S, Narayana B, Byrappa K, Wang Y, Yao D, Sangappa Y (2015) Gamma radiation assisted biosynthesis of silver nanoparticles and their characterization. Adv Mater Lett 6:1088-1093

31. Narimani M, Teimouri A, Shahbazara Z (2019) Synthesis, characterization and biocompatible properties of novel $11 \mathrm{oin} / g r a p h e n e$ oxide nanocomposite scaffolds for bone tissue engineering application. Polym Bull 76:725-745

32. Shanmugam M, Alsalme A, Alghamdi A, Jayavel R (2016) In-situ microwave synthesis of graphene$\mathrm{TiO}_{2}$ nanocomposites with enhanced photocatalytic properties for the degradation of organic pollutants. J Photochem Photobiol B 163:216-223

33. Wang SD, Ma Q, Wang K, Chen HW (2018) Improving antibacterial activity and biocompatibility of bioinspired electrospinning silk fibroin nanofibers modified by graphene oxide. ACS Omega 3:406-413

34. Geng D, Yang S, Zhang Y, Yang J, Liu J, Li R, Sham T-K, Sun X, Ye S, Knights S (2011) Nitrogen doping effects on the structure of graphene. Appl Surf Sci 257:9193-9198

35. Bo Liang Lu, Fang YH, Yang G, Zhu Q, Ye X (2013) Fabrication and application of flexible graphene silk composite film electrodes decorated with spiky Pt nanospheres. Nanoscale 00:1-3 
36. Gianak O, Pavlidou E, Sarafidis C, Karageorgiou V, Deliyanni E (2018) Silk fibroin nanoparticles for drug delivery: effect of bovine serum albumin and magnetic nanoparticles addition on drug encapsulation and release. Separations 5:25. https://doi.org/10.3390/separations5020025

37. Zhang X, Bao H, Donley C, Liang J, Yang S, Shui Xu (2019) Thiolation and characterization of regenerated Bombyx mori silk fibroin films with reduced glutathione. BMC Chem 13:62. https://doi.org/10. 1186/s13065-019-0583-x

38. Liu J, Cui L, Kong Na, Barrow CJ, Yang W (2014) RAFT controlled synthesis of graphene/polymer hydrogel with enhanced mechanical property for pH-controlled drug release. Eur Polym J 50:9-17

39. Yang Yi, Ding X, Zou T, Peng Ge, Liu H, Fan Y (2017) Preparation and characterization of electrospun graphene/silk fibroin conductive fibrous scaffolds. RSC Adv 7:7954-7963

40. Lia H, Zhanga Z, Godakanda VU, Chiu YJ, Angkawinitwong U, Patel K, Stapleton PG, de Silva RM, de Silva KMN, Zhu LM, Williamsa GR (2019) The effect of collection substrate on electrospun ciprofloxacin-loaded poly (vinylpyrrolidone) and ethyl cellulose nanofibers as potential wound dressing materials. Mater Sci Eng C 104:109917

41. Kooti M, Sedeh AN, Motamedi H, Rezatofighi SE (2018) Magnetic graphene oxide inlaid with silver nanoparticles as antibacterial and drug delivery composite. Appl Microbiol Biotechnol 102:3607-3621

42. Cacicedo ML, Pacheco G, Islan GA, Alvarez VA, Barud HS, Castro GR (2020) Chitosan-bacterial cellulose patch of ciprofloxacin for wound dressing: preparation and characterization studies. Int J Biol Macromol 147:1136-1145

43. Gasymov OK, Aydemirova A, Alekperov O, Aslanov RB, Khalilova K, Gasimov N, Mamedov N, Mamedova I, Babayev S, Gasanov N (2015) IR ellipsometry of silk fibroin films on Al nanoislands. Phys Status Solidi C. https://doi.org/10.1002/pssc.201500005

44. Johra FT, Lee JW, Jung WG (2014) Facile and safe graphene preparation on solution based platform. J Ind Eng Chem 20:2883-2887

45. Wang S, Ning H, Ning Hu, Huang K, Weng S, Xiaopeng Wu, Liangke Wu, Liu J (2019) Preparation and characterization of graphene oxide/silk fibroin hybrid aerogel for dye and heavy metal adsorption. Compos B Eng 163:716-722

46. Wang F, Aravind SSJ, Wu H, Forys J, Venkataraman V, Ramanujachary K, Hu X (2017) Tunable green graphene-silk biomaterials: mechanism of protein-based nanocomposites. Mater Sci Eng C 79:728-739

47. Gao H, Sun Y, Zhou J, Rong Xu, Duan H (2013) Mussel-inspired synthesis of polydopamine-functionalized graphene hydrogel as reusable adsorbents for water purification. ACS Appl Mater Interfaces 5:425-432

48. Chang S-Q, Dai Y-D, Kang B, Han W, Chen Da (2009) Gamma radiation synthesis of silk fibroin coated CdSe quantum dots and their biocompatibility and photostability in living cells. J Nanosci Nanotechnol 9:5693-5700

49. Huang T, Zhang L, Chena H, Gao C (2015) Cross-linking graphene oxide-polyethyleneimine hybrid film containing ciprofloxacin: one-step preparation, controlled drug release and antibacterial performance. J Mater Chem B. https://doi.org/10.1039/C4TB01896F

50. Najafabadi SAA, Mohammadi A, Kharazi AZ (2020) Polyurethane nanocomposite impregnated with chitosan-modified graphene oxide as a potential antibacterial wound dressing. Mater Sci Eng C 115:110899

51. Han F, Dong Y, Song A, Yin R, Li S (2014) Alginate/chitosan based bi-layer composite membrane as potential sustained-release wound dressing containing ciprofloxacin hydrochloride. Appl Surf Sci 311:626-634

52. Ma R, Wang Y, Qi H, Shi C, Wei G, Xiao L, Huang Z, Liu S, Haipeng Yu, Chunbo Teng Hu, Liu VM, Zhang J, Wang Y, Guo Z (2019) Nanocomposite sponges of sodium alginate/graphene oxide/polyvinyl alcohol as potential wound dressing: in vitro and in vivo evaluation. Compos B 167:396-405

53. Li X, Liu Y, Zhang J, You R, Jing Qu, Li M (2017) Functionalized silk fibroin dressing with topical bioactive insulin release for accelerated chronic wound healing. Mater Sci Eng C 72:394-404

Publisher's Note Springer Nature remains neutral with regard to jurisdictional claims in published maps and institutional affiliations. 